\title{
LIQUID CRYSTALLINE ACRYLATES AND THEIR POLYMERS
}

In previous papers $(1,2)$ the thermotropic properties and the kinetics of the bulk polymerization of the mesomorphic monomer cholesteryl acrylate have been described. In addition to cholesteryl acrylate, the methacrylate was prepared, as well as the respective acrylic methacrylic acid ester of cholestanol. We now wish to report the properties of these monomeric esters and the results of their polymerization.

The esters were synthesized according to the method described in ref. (2). Cholesteryl acrylate and methacrylate were chemically pure after repeated crystallization from ether/ethanol. The cholestanyl esters had to be further purified by preparative thin layer chromatography. Kiesel gel GF 254 was applied as the adsorbent, light petroleum ether $60-80^{\circ} \mathrm{C} /$ isopropyl ether $(50 / 1)$ was used as developer. The esters after elution with chloroform and addition of an equal volume of ethanol were precipitated at $4^{\circ} \mathrm{C}$.

Elemental analysis of the four monomers yielded the percentages summarized in Table I.

The thermotropic properties of the esters were determined by means of differential thermal analysis (Du Pont DTA, model 900), by observation through a hot-stage microscope, equipped with nicols, and by $x$-ray diffraction (GuinierLenné high-temperature camera). The transition temperatures, including those of cholesteryl acrylate are presented in Table II.

The temperatures shown in Table II were determined during the first heating or cooling cycle. After repeated heating and cooling the transition temperatures started to differ from the original values due to formation of polymer. In several cases a phase separation between polymer and monomer was observed under the microscope.

The thermotropic properties of the respective monomers, except cholesteryl acrylate of which the properties have been described earlier (2), will be discussed hereafter.

Cholesteryl methacrylate exhibits a monotropic mesophase. The solid needles melt forming an isotropic liquid at $114^{\circ} \mathrm{C}$ as observed under the microscope. When the sample is cooled, supercooling occurs and at $111.5^{\circ} \mathrm{C}$ the mesophase, having the focal-conic texture, is formed. Crystallization occurs at approximately $92^{\circ} \mathrm{C}$ on further cooling. The solid-solid transition at $64^{\circ} \mathrm{C}$ does not take place in crystals formed from the melt. According to Tanaka et al. (3) the mesophase of cholesteryl methacrylate is enantiotropic. We found however that crystals formed from solution as well as from the melt directly transform to the isotropic liquid phase. Saeki et al. (4) also observed that the mesophase was monotropic but they reported transition temperatures 6 to 8 degrees lower than the temperatures given in Table II. 


\section{TABLE I}

Elemental Analysis of the Steroid Esters

\begin{tabular}{|c|c|c|c|c|}
\hline & $\begin{array}{c}\% \mathrm{C} \\
\text { Found Calc. }\end{array}$ & $\begin{array}{l}\% \mathrm{H} \\
\text { Found Calc. }\end{array}$ & Foun & $\begin{array}{l}\mathrm{O} \\
\mathrm{d} \text { Calc. }\end{array}$ \\
\hline $\begin{array}{l}\text { Cholesteryl acrylate } \\
\mathrm{C}_{30} \mathrm{H}_{48} \mathrm{O}_{2}\end{array}$ & $81.8 \quad 81.76$ & $\begin{array}{ll}11.0 & 10.98\end{array}$ & 7.3 & 7.26 \\
\hline $\begin{array}{l}\text { Cholesteryl methacrylate } \\
\mathrm{C}_{31} \mathrm{H}_{50} \mathrm{O}_{2}\end{array}$ & $81.8 \quad 81.87$ & $11.0 \quad 11.09$ & - & 7.04 \\
\hline $\begin{array}{l}\text { Cholestanyl acrylate } \\
\mathrm{C}_{30} \mathrm{H}_{50} \mathrm{O}_{2}\end{array}$ & $81.6 \quad 81.40$ & $11.8 \quad 11.38$ & 6.7 & 7.22 \\
\hline $\begin{array}{l}\text { Cholestanyl methacrylate } \\
\mathrm{C}_{31} \mathrm{H}_{52} \mathrm{O}_{2}\end{array}$ & $81.3 \quad 81.50$ & $11.8 \quad 11.49$ & 7.1 & 7.01 \\
\hline
\end{tabular}

Cholestanyl acrylate exhibits a mesophase between $98^{\circ} \mathrm{C}$ and $104^{\circ} \mathrm{C}$, as determined by differential thermal analysis, and between $96.5^{\circ} \mathrm{C}$ and $104^{\circ} \mathrm{C}$ as observed under the microscope. During the first heating the material forms the Grandjean plane texture. When the sample is cooled from the isotropic liquid phase, the mesophase that again occurs at $104.5^{\circ} \mathrm{C}$ adopts the focalconic texture like the mesophase of the other monomers. The cholesteric focal-conic texture is also formed from crystals from the melt.

Cholestanyl methacrylate exhibits a monotropic, cholesteric mesophase. The solid-liquid transition takes place at $95^{\circ} \mathrm{C}$, determined by DTA and microscopy. On cooling the transition into the mesophase occurs at $85^{\circ} \mathrm{C}$ and crystallization sets in at ca. $73^{\circ} \mathrm{C}$.

Summarizing, we see that the temperature ranges of the mesophase of the four monomers are narrower than those of cholesteryl propionate $\left(102-116^{\circ} \mathrm{C}\right)$ (5) and cholesteryl butyrate $\left(102-113^{\circ} \mathrm{C}\right)(5)$, which have the same number of carbon atoms as cholesteryl acrylate and methacrylate, respectively. In particular, the solid-mesophase transition has shifted to a temperature higher than that of the aliphatic esters. The solid state is possibly more stable because the double bond of the acrylic group enhances the terminal attraction forces between the molecules. The occurrence of a smectic mesophase would not have been surprising in this case for the same reason. Substitution of the $\alpha \cdot \mathrm{H}$ atom of the acrylic group by methyl lowers the temperature of the solid-mesophase transition by about 10 degrees. Conceivably the terminal attraction between the methacrylic groups becomes less by the steric interaction between the branched groups.

Elimination of the $\Delta 5,6$ double bond in the cholesteryl moiety has a downward effect on the transition temperatures of about 25 degrees. This can be the result of the lesser polarizability of the cholestanyl esters. In smectogenic 
TABLE II

Thermotropic Properties of the Monomers

\begin{tabular}{lccc}
\hline & \multicolumn{3}{c}{ Temperature of Transition $\left({ }^{\circ} \mathrm{C}\right)$ to phase } \\
\cline { 2 - 4 } & solid-solid & cholesteric & isotropic \\
\hline Cholesteryl & $65^{\mathrm{a}}$ & $121^{\mathrm{a}}$ & $126^{\mathrm{a}}$ \\
Acrylate & $64^{\mathrm{c}}$ & $122.5^{\mathrm{b}}$ & $125^{\mathrm{b}}$ \\
Cholesteryl & $64^{\mathrm{a}}$ & & $115^{\mathrm{a}}$ \\
Methacrylate & $64^{\mathrm{c}}$ & {$[111.5]^{\mathrm{d}}$} & $114^{\mathrm{b}}$ \\
Cholestanyl & & $98.0^{\mathrm{a}}$ & $104^{\mathrm{a}}$ \\
Acrylate & & $96.5^{\mathrm{b}}$ & $104.5^{\mathrm{b}}$ \\
Cholestanyl & & & $95^{\mathrm{a}}$ \\
Methacrylate & & {$[85]^{\mathrm{d}}$} & $95^{\mathrm{b}}$ \\
\hline
\end{tabular}

aDetermined by DTA in $\mathrm{N}_{2}$ atmosphere at $2 \mathrm{deg} / \mathrm{min}$ heating rate.

bDetermined by microscopy, heating rate: about $1 \mathrm{deg} / \mathrm{min}$.

cDetermined by $\mathrm{x}$-ray diffraction in $\mathrm{N}_{2}$ atmosphere at $0.02-0.03 \mathrm{deg} / \mathrm{min}$ heating rate.

$\mathrm{d}_{\text {Numbers inside brackets are the transition temperature of a monotropic }}$ mesophase, microscopically determined on cooling (rate: approximately 1 $\mathrm{deg} / \mathrm{min})$.

and nematogenic compounds, the presence of a double bond generally enhances mesophase stability. Gray (6) points out that the effect of the double bond in the sterol skeleton on the stability of the cholesteric-isotropic transition tends to be lower if the double bond is absent.

All four monomers readily polymerized in bulk to insoluble products when heated in air above their mesomorphic-isotropic transition temperature. Soluble polymers were obtained when the thermal polymerization was carried out in vacuo as described in ref. (2). Solvents were tetrahydrofuran, benzene, toluene, and methylene chloride. Solution polymerization in benzene, using benzoyl peroxide as initiator, yielded conversions of about $80 \%$. The molecular weights of these polymers determined by ultracentrifugation were in the order of 3 to $8 \times 10^{5}$. None of the polymers showed any liquid crystallinity when swollen or dissolved by a solvent.

The results of our study on the effect of monomer preorientation in the cholesteric mesophase on polymer structure will be given in a forthcoming paper. 


\section{References}

(1) A. C. de Visser, J. Feyen, K. de Groot, and A. Bantjes, J. Polym. Sci., B, $\underline{8}, 805$ (1970).

(2) A. C. de Visser, K. de Groot, J. Feyen, and A. Bantjes, J. Polym. Sci., A-1, $\underline{9}, 1893$ (1971).

(3) Y. Tanaka, S. Kabaya, Y. Shimura, A. Okada, Y. Kurihara, and Y. Sakakibara, J. Polym. Sci., B, 10, 261 (1972).

(4) H. Saeki, K. Iimura, and M. Takeda, Polym. J., $\underline{3}$, 414 (1972).

(5) G. W. Gray, J. Chem. Soc., 1956, 3397 (1956).

(6) G. W. Gray, "Molecular Structure and the Properties of Liquid Crystals," Academic Press, London, 1962, p. 192.
A. C. de Visser
K. de Groot

Dept. of Material Sciences

School of Medicine and Dentistry

Free University

Amsterdam, The Netherlands

Polymer Division

Dept. of Chemical Technology

Twente University of Technology

Enschede, The Netherlands

Received September 22, 1972 